Wird nun diese blaustichige Masse 3 bis 4 mal durch Dekantieren mit destilliertem Wasser gewaschen, dann verteilt sich der Niederschlag im Waschwasser, ohne sich wieder abzusetzen, und geht dann in wenigen Stunden voll. komrien in Lösung über. Es resultiert also die kolloidale Lösung des weißen Zinnoxysulfides in Wasser: Diese reagiert auf Lackmus sauer und Säuren bewirken in ihr keine Fällung. Daraus darf aber im Verein mit den übrigen Beobachtungen der Schluß gezogen werden, daß die kolloidale wässerige Lösung die zweibasische Säure, $\mathrm{Sn}_{2} \mathrm{~S}_{3} \mathrm{O} . \mathrm{H}_{2} \mathrm{O}$, von der Konstitution:

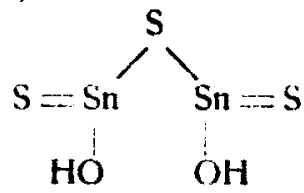

enthălt. Gleich dem Verhalten aller anderen kolloidalen Lösungen, werden auch in dieser durch Neutralsalze, wie Kochsalz, Natrium. acetat usw. Fällungen hervorgerufen. Ueber die spezifisch kolloidale Natur des weißen Zinnoxysulfides kann daher kein Zweifel mehr aufkoinmen.

Dem weißen, aumonkarbonatlöslichen Zinnoxysulfid selbst kommtdieKonstitution:<smiles>S=[Sn]1O[Sn]1S</smiles>

zu, während Anzeichen dafür vorliegen, daß die ursprüngliche Fällung das saure Ammonium. salz:

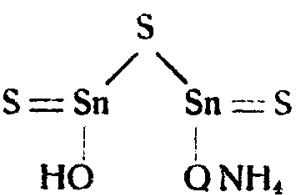

\title{
Die wichtigsten Fragen der Chemie der Kolloide.
}

Von P. D. Zacharias in Athen und B. Kuriloff in Jekaterinoslaw.

$\$ 1$. Ueber den Zweig der Chemie, der die Bezeichnung Kolloidalchemie führt und dessen Bedeutung nunmehr von allen Seiten gebührend anerkannt wird, sind wir noch so ziemlich im Dunkeln, die gebräuchlichen Bezeichnungen und Definitionen sind einer großen Anzahl Fachgenossen nicht genug klar. Beim sechsten internationalen Kongreß für angewandte Chemie in Rom im Mai dieses Jahres ist dieser Teil der Chemie zum Gegenstand von Vorträgen und Diskussionen gemacht worden. Die Verfasser dieses Aufsatzes haben die Vorträge "Zur Chemie der Farblacke" (Zacharias) und "Ueber den Uebergang von kristallinischen zu kolloidaien Körpern (Kuriloff) gehalten. Es war auch eine allgemeine Diskussion über die Kolloide angeregt worden, welche aber nicht zustande kam, dem die Herren, welche sie angeregt hatten und die zu diskutierenden Punkte feststellen sollten, waren nicht erschienen und haben auch ihre Wünsche nicht schriftlich mitgeteilt, so dab man zur Annahme gezwungen wird, daß sie wohl eine Diskussion zur Schaffung von Klarheit wünschten, aber keine Zeit dazu hatten, wenn man die eine Annahme ausschließt, daß sie sich selbst nicht klar waren, wie dieselbe einzuleiten und durchzuführen wäre.

Mit der Chemie des Kolloidalzustandes seit längerer Zeit beschäftigt und von der Wichtig. keit der Aufstellung eines Diskussionsplanes zur Förderung der Kolloidalchemie und Verbreitung der schon gewonnenen zahlreichen Erfahrungen fest überzeugt, sind wir in Rom an Schlufs des Kongresses nach einer längeren Besprechung der Angelegenheit zu der weiter unten präzisierten Kennzeichnung der wichtigsten Punkte der Kolloidalchemie gekommen und übergeben wir dieselbe der Oeffentlichkeit in der Hoffnung damit eine gewisse Bestimmtheit in der Kennzeichnung des Kolloidalzustandes einzuführen, soweit es gegenwärtig möglich ist, klare Vorstellungen zu verbreiten und das Studium dieses neuen und so wichtigen Chemiezweiges zu fördern.

Die weiter unten bestimmten Punkte stammen zum größten Teil aus den eigenen Arbeiten über die Färbevorgänge und die Absorption einerseits ') und aus den Studien der Ammoniakate andererseits ${ }^{2}$.

1) P. D. Zacharias. Sur la théorie de la teinture: IV. internationaler Kongreß $\mathrm{f}$. ang. Chemie, Paris 1900 ;

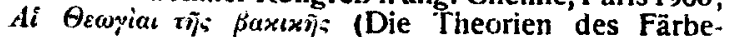
vorganges), Athen 1900; Ueber den Zustand und die Eigenschaften der Kolloide, Z. f. phys. Chemie 39, 468; Gerben mit Farbstoffen, V. intern. KongreB. f.' ang. Chemie, Berlin 1903; Zur Chemie der Textilfasern, Z. f. Farben u. Textilchemse 1903, Heft 12; Adsorption oder Absorption, Chem. Zeitung 1905, Nr. 39.

2) B. Kuriloff. Sur la théorie des ammoniakates, Ann. Chem. Phys. 1906, 8. Serie, 8, 518; Populäre Einleitung in das Studium d. Naturwissenschaften 
Es wurden im ganzen 12 Punkte hervorgehoben; dieselben dürfen nicht als auf alle Fälle anwendbar betrachtet werden. Es sei betont, dab mehrere Arten von Kolloiden existieren, welche auch für sich besondere Eigenschaften zeigen, die im allgemeinen mit denen der anderen Gruppen nicht ganz übereinstimmen. Es würde aber wohl verfrüht sein, wenn man eine Teilung jetzt vornehmen wollte und getrennte Metallhydrosole, Oxydhydrosole, organische Kolloide usw: behandelte. Wir werden vielmehr auf das ganze Gebiet Bezug nehmen und entscheidende Momente anführen, die der einen oder anderen Gruppe besonders eigen sein mögen, die aber zur scharfen Kennzeichnung des Kolloidalzustandes im allgemeinen geeignet sind und seine allgemeinen Eigenschaften ausmachen. Es wird hoffentlich auf viele lange bestehende Streitfragen segensreich wirken, wenn das Vorhandensein des Kolloidalzustandes-erkannt oder ausfindig gemacht werden kann, weil in diesem Falle die ganze Behandlung des Problems weit verschieden sein wird und die SchluBfolgerungen auf andere Weise erfolgen können, als wenn nur Wechselwirkungen von Körpern in kristallinischem Zustande ins Spiel kommen. Die Verfasser haben demzufolge die Klassifikationsirage der verschiedenen Kolloide außer Betracht gelassen ${ }^{3}$ ).

5 2. Die in Frage kommenden Punkte der Kolloidalchemie sind nunmehr folgende:

1. Eine kolloidale Lösung ist als eine Pseudolösung zu betrachten.

Sie ist insofern als eine wirkliche Lösung anzusehen, als man unter dem Begriff "Lösung * eine klare, homogene Zerteilung des gelösten Körpers versteht $)$. Optisch nach dem Tyndallschen Phänomen untersucht, zeigt sie sich inhomogen. Bei der Pseudolösung fällt der Begriff der Sättigung der Lösung weg.

2. Scheinbare Abscheidung des festen Körpers ist als totale oder partielle Koagulation zu betrachten.

Totale Koagulation tritt bei einem bestimmten

(Verlag von Krapp) 1906, die belebte Natur; Ueber den Uebergang von kristallinischen zu kolloidalen Körpern, Z. f. Electrochemie, 12, 209; Sur la theorie des ammoniakates en relation avec le problème de la classification chimique génerale (russisch) Jekaterinoslaw 1905.

) Vergl. Müller (Z. anorg. Chemie 36, 340). Duclaux C. R. 138, 144. Bibllographie der Kolloide. Müller. Z. anorg. Chemie 39, 12

4) In diesem Sinn können die Resultate der Arbeit von D. P. Konowaloff itber Opalescenz Uournal Russ. Chem. Oes. 1902, 738) interpretiert werden.
Wert der inneren Reibung ${ }^{8}$ ) ein. Die kolloidalen Körper können zwar durch verschiedene Umstände zur Koagulation gebracht werden, doch spielt die Konzentration der Pseudolösung dabei so gut wie keine Rolle. Ein Sol kann durch Schütteln in ein Gel übergehen, also durch eine mechanische.Kraft, oder auch durch $\mathrm{Er}$ hitzen oder durch andere Umstände dazu veranlaBt werden. Die totale Koagulation kommt bei den Systemen vor, die aus hoch komplexen Molekülen erbaut sind (Gelatine, Gummi, Silikate, komplexe Ammoniakverbindungen usw.).

3. Partielle Koagulation heiBt die Entmischung (Sedimentation) der scheinbar homogenen Lösung in zwei scheinbar inhomogene Phasen.

Partielle Koagulation tritt beim Zusatz von Elektrolyten sowohl als auch von kolloidalen Stoffen und anderen Substanzen (Alkohol usw.) ein. Partielle Koagulation kann zwar durch dieselben Faktoren wie totale Koagulation verursacht werden, doch tritt sie alich bei den Systemen ein, die aus einfachen Molekülen erbaut sind (Metallhydrosole $u$. dergl.). Diese Fällung hat ihren Grund nicht in der Veränderung der Natur des Lösungsmittels, sondern in der Veränderung des Zustandes der kolloidal gelösten Substanz.

4. Der Dampfdruck einer kolloidalen Lösung ist gleich oder fast gleich dem des Lösungsmittels.

Daraus folgt, daB Siedepunktserhöhung und Gefrierpunktserniedrigung sehr klein oder fast gleich Null sind. Jedoch zeigt eine kolloidale Lösung eine Art pseudoos motischen Drucks, der als Triebkraft bei der freien Diffusion des kolloidal gelösten Körpers wirkt.

5. Die Leitfähigkeit einer kolloidalen Lösung ist sehr klein, wenn auch große Mengen Substanz gelöst sind.

Diese Leitung ist vielmehr eine Pseudoleitung, indem sie konvektiver Natur ist, $d . h$. die wardernden Teilchen sind keine lonen im gewöhnlichen Sinne, sondern ganze Moleküle oder Molekülaggregate.

6. Oewöhnliche Reaktionen sind beim Kolloidalzustand ausgeschlossen.

Es fehlt an einer bestimmten Reaktionsgröße, wie es beim kristallinischen Zustande das $\mathrm{Mol}$ ist. Bei Wechselwirkungen zwischen Kolloiden miteinander oder mit Kristalloiden treten keine bestimmten Verhăltnisse auf. Gewöhnliche

\% Vergl. Arbeit von S. Lewites (Journ. Russ. Chem. Oes. 1904, 401). 
chemische Formeln der Verbindungen und chemische Gleichungen versagen ihren Dienst. Erst wenı das Kolloidalgebilde zerstört wird, treten die gewöhnlichen Reaktionen zutage.

7. Das Verteilungsgesetz im Sinne von Berthelot-Nernst ist nicht anwendbar.

Aus der Verteilungsgleichung können keine Schlüsse auf die MolekulargröBe der beteiligten Körper gezogen werden, wenn sich auch der verteilte Körper im kristallinischen Zustande befindet.

8. Das Massenwirkungsgesetz in seiner gegenwärtigen Formulierung und die Phasenregel nach Gibbs-Rooseboom sind nicht anwendbar.

Das Gesetz der Massenwirkung ist auf Grund gewisser molekulartheoretischer und kinetischer Betrachtungen entwickelt worden. Als wirksame Masse ist immer die Zahl der noch vorhandenen Mole zu verstehen und das Vorhandensein von Körpern mit einer bestimmten Reaktionsgröße sowie die Reaktion nach einer bestimmten Formel sind Bedingungen. der Anwendbarkeit des Gesetzes. Das trifft aber beim Kolloidalzustande nicht zu.

Die Phasenregel andererseits frei von Molekularbetrachtungen ist einer viel allgemeineren Anwendbarkeit fähig. Jedoch ist sie auch auf Systeme beschränkt, für deren Zustand nur Temperatur, Druck und Konzentration der Bestandteile maBgebend sind. Beim Kolloidalzustande kommen noch andere Faktoren in Betracht; Oberflächenenergie (Ostwald), kapillarelektrische Phänomene (Bredig), zeitliche Aenderung der Eigenschaften (van Bemmelen, Kuriloff) usw. Sobald in einem System ein Gel ausgeschieden wird, nimmt es durch seinen Koagulationszustand und den dabei durch Volumänderungen hervorgerufenen Kräften an dem Gleichgewicht des Systems einen hervorragenden Anteil (Zacharias).

9. Bei der Absorption von gelösten Körpern durch Kolloide wird der Oleichgewichtszustand durch die Gleichung $\frac{c}{c_{1}{ }^{\nu}}=a$ ausgedrückt, wenn $c-$ die Konzentration der Lösung, $c_{1}$ - die Konzentration des absorbierenden Mediums, ${ }^{\nu}$ - und $\alpha$ Konstanten sind, unter der Voraussetzung, daß die beteiligten Körper ihre Natur nicht ändern.

Aendert bei der Absorption der absorbierende Körper sein Volum durch Quellung, dann ist das Teilungsverhältnis nicht konstant, sondern eine Funktion der Endkonzentration $\frac{c}{c_{1}}=F(K)$ (van Bemmelen) ${ }^{8}$, wobei $\mathrm{K}$ im allgemeinen mit der Zunahme der Konzentration stark abnimmt (Zacharias).

Die Konstanz des Wertes des Koeffizienten $v$ gibt uns ein Maß der Rigidität des absorbierenden Mediums. Sobald der absorbierende Körper sein Volum ändert (durch Quellung), bekommt fortwährend die Größe $y$ einen anderen Wert.

Wenn der Gang der Absorption durch eine Kurve dargestellt wird mit $c$ als Ordinate und $c_{1}$ als Abscissen, dann nähert sich je größer die Quellung wird die Kurve einer horizontalen Linie, welche uns den Zustand der Lösung des Kolloids darstellt, wobei die Konzentration ihren Grenzwert erreicht hat und die VolumenvergröBerung gleichen Schritt mit der aufgenommenen Menge hält.

10. Der kolloidal gelöste Körper kann durch ein Kolloid vollstăndig aus der Lösung entfernt werden.

Wenn der absorbierte Körper kolloidal ist, dann verliert die oben angegebene Absorptionsgleichung wegen eintretender Koagulation ihre Bedeutung.

Die totalen Ausscheidungen der kolloidal gelösten Substanzen können chemische Präzipitationen vortäuschen.

11. Zeitliche Aenderung der Eigenschaften ist ein Grundmerkmal des Kolloidalzustandes.

Das Alter der koagulierenden (oder kolloidal gelösten) Substanzen ist von empfindlichem EinfluB auf ihre Eigenschaften.

12. Kontinuität der Eigenschaften ist das prägnanteste Charakteristikum des Kolloidalzustandes.

Zusammensetzung und Zustand überhaupt des Körpers sind Funktionen der äuReren Bedingungen (Temperatur, Konzentration, Zusammensetzung des umgebenden Mediums usw.) und ändern sich stetig mit denselben.

So können die Körper im Kolloidalzustand durch keine chemische Formel in ihrer Zusammensetzung definiert werden, sondern durch die Gleichung des Absorptionsgleichgewichts oder durch die entsprechende Kurve. Die gebildeten Verbindungen sind Absorptionsverbindungen (van Bemmelen) zu nennen, welchen eine gewisse mehr oder weniger ausgeprägte Inhomogenität in Bau anhaftet und als Molekularverbindungen (Additionsprodukt zweiter Klasse nach Kuriloff) angesehen werden könuen.

\footnotetext{
B) van Betnmelen, Zn anorg. Chem. 23, 32I.
} 
Die beiden letzten Merkmale charakterisieren den Kolloidalzustand als einen dynamischen Zustand. Die Körper erleiden dabei stetige Zustandisändcrungen, bis sie zur stabilen Gleichgewichtslage des kristallinischen Zustandes kommen. Die Eigenschaften des kristallinischen Zustandes sind die Grenzyebiete, welche wirklich oder asymptotisch erreicht werden. Ist das yeschehen, dann ist der Körper aus dem Kolloidalzustand heransgetreten.

Im Folgenden wollen wir die oben angegebenen Punkte womöglich an der Hand bestimmter typischer Beispiele von Kolloidalgebilden und praktischer Anwendungen erläutem.

§ 3. Das schönste Beispiel einer kolloidalen Lösung mit den einfachsten Eigenschaften ist eine Lösung von metallischem Gold, oder das Goldhydrosol. Diesesläft sich nach Bredig? durch Kathodenzerstäubung in einer Konzentration von $0.014 \mathrm{~g}$ auf $100 \mathrm{ccm}$ und mach Zsigmondy ${ }^{8}$ ) durch Reduktion der Goldlösung mit Formaldehyd oder Phosphor in einer Konzentration von 0.005 Proz. bereiten. Es stellt eine klare homogene Flïssigkeit von tief purpurroter Farbe dar und ist eine äuBerst feine Zerteilung von metallischem Golde, jedoch keine Suspension. Erst durch das Tyndall'sche Phänomen (Ultramikroskopie nach Zsigmondy) wird eine Inhomogenität in der Löstnig entdeckt. Die Teilchen sind kleiner als $140 \%$ die Grenze der Sichtbarkeit in Mikroskop. Zsigmondy hat gefunden, daß Goldteilchen von $75 \%$ Größe bereits absetzen, so da? die beständigen Goldhydrosole Teilchen von der Größe 60-6 $\eta^{\prime \prime}$ enthalten, also ziemlich nahe an die sogenannte Molekulardimension von $0.10 \mathrm{z \prime \prime}$. Die Farbe der Lösung ändert sich mit der Zeit und geht in bläulich und violett iber, was den Beginn der Zusammenflockung anzeigt. Thre Filtrierbarkeit hängt von der Teilchengröße ab. Die feinsten Sole gehen auch durch porösen Ton unverändert durch. Bein Durchleiten des elektrischen Stromes wandert das Gold zur Anode und scheidet sich dort ais ein schwarzes metallisches Pulver aus. Durch Ausfieren der Lösung wird das Gold knaguliert; ebenso durch die kieinsten Zusätze von Elektrolyten: Säuren wirken dabei bedeutend sclineller als Basen, welche nur bei großer Konzentration fällen. In sehr kleinen Mengen wirken die Basen sogar schützend und die feinsten Sole sind in der Tal in schwach alkalischen Medien

7) Anorganische Fermente 1901, Leipzig.

3) Zut Erkenntmis der Kolloide, Jena 1905. gewonnen worden. Kolloide wirken sehr. verschieden: manche fällen das Gold, andere schützen es. Der Vorgang der Koagulation ist beim Gold irreversibel: das einmal gefällte Gold läßt sich nicht mehr einfach mit Wasser wieder in das Sol bringen. Gelatine, welche schützend wirkt, kann den Vorgang irreversibel machen: also bei der Koagulation und Wiederlösung der Gelatine wird das Gold in den Niederschlag und dann in die Lösung mitgerissen. Aehnlich wirkt auch Zinnoxyd im Cassiuspurpur, welcher ein Gemisch von Zinnoxyd- und Goldhydrosol ist (Zsigmondy). Eine Fuchsinlösung in Wasser, welche kolloidal ist, fallt das Gold aus dem Sol: aus dem Niederschlag läßt sich das Fuchsin durch Wasser nicht auswaschen, wohl aber durch Alkohol. Durch kolloidale Tonerde wird das Gold aus dem Sol entfernt unter Bildung eines purpurroten Lackes, der ähnlich wie der Karminlack aussieht. Wir sehen, daß Gold je nach seinem Alter und Vorbereitungsart verschiedene Farbe and Filtrierbarkeit besitzt. Es reagiert im gewöhnlichen Sinne nicht. Das Fallen des Goldes ohne jeglichen Zusatz oder die Bildung von Absorptionsverbindungen ohne bestimmte Verhälinisse treten nicht in das Gebiet der gewöhnlichen chemischen Reaktionen. Am kolloidalen Silber sehen wir sehr gut die Unfähigkeit der Kolloide zu gewölunichen Reaktionen. Ein Silbersol wird durch Salpetersäure zuerst koaguliert: erst nach einiger Z.eit, nachdem die Kolloidalmoleküle zerstört worden sind, tritt Lösung unter Gasentwicklung ein.

$\$ 5$. Ein anderes typisches Beispiel von Kolloid besonderer Gattung ist Gelatine (Gummi usw.). Hier haben wir Körper von komplexer Zusammensetzung vor uns, was wohl der allgemeinere Fall ist. Gelatine ist eine amorphe Substanz von verschiedener Weichheit ie nach Wassergehalt und Temperatur. In Wasser quillt sie auf und bei gecigneter Temperatur löst sie sich. Die Quellung ?) ist verschieden in Wasser oder in einer Atmosphäre von gresāttigtem Wasserdampf. Die L.ösurg ist klar, farblos, mit grober innerer Reibung und gelatiniert leicit. Sie ist optisch inhomogen (ultramikroskopisch) wie das Goldhydrosol. Die Gelatinierung der Lösung ist als totale Koagulation des komplexen Gemisches zu betrachten. Diese wird durch Zusatz von Sulfaten der Alkalien befördert, durch Chloride und Nitzale verzögert. Die innere Reibung der

9) Patil ron Schroder. Ueber Erstarrungs- und Quellungserscheinungen der Gelatine. Z. f. phys. Ch. 45, 75-117. 
Lösung ist ein Maß des Zustandes derselben und ist bei gegebener Temperatur keine Konstante, sondern von der chemischen Vorgeschichte der betreffenden Losung abhängig, indem bein $\mathrm{Ab}$ kühlen einer erhitzten Lösung, nachdem die anfängliche Temperatur wieder erreicht worden ist, der Wert der inneren Reibung langsamer zum ursprïnglichen Wert zurückkehrt. Bei dcr Gelatine wie bei vielen komplexen Kolloiden tritt eine allmāhliche Wirkung des Wassers' auf, wodurch die Substanz irreversibel verändert und die einfachen Vorgänge verwickelt werden. Durch großie Salzzusätze wie auch durch Alkohol und andere Mittel tritt eine Entmischung des Gels ein. Der Vorgang ist eine wahre Koagulation, indem er nicht von der Aenderung des Zustandes des Lösungsmittels abhängt, sondern in der Aenderung des Quellungs- und Wasserbindevermögens des Kolloides seinen Grund hat. Eine 50 proz. Gelatinelösing siedet bei $99.8^{\circ}$ nach Lüdeking. Der osmotische Druck einer $6 \mathrm{proz}$. Gelatinelösung ist von I'feffer $z u$ $21,3 \mathrm{~cm}$ Quecksilber gefunden. worden, was einem Molekulargewicht von 5200 entsprechen würde. Bestimmte Reaktionen der Gelatine sind nicht bekannt. Säuren und Alkalien wirken zersetzend: die Geschwindigkeit dieser Umwandlung ist abhängig von der Temperatur, der Konzentration der Gelatinelösung und von chemischen Zusätzen. Die Säuren wirken dabei katalytisch, inden ihre Totalmenge unverändert bleibt.

Der Vorgang der Koagulation der Gelatine ist auch in der Natur und Technik von Bedeutung. Die Fasern der Hautsubstanz bestehen aus Gelatine in einem vorgeschrittenen Zustand der Koagulation, nicht aber soweit, daß sie durch Wasser nicht wieder in Lōsung gebracht werden können. Durch verschiedene koagulierende Mittel werden sie ihres großen Wasserbindevermögens beraubt und so zu dem nicht faulenden geschmeidigen Oebilde, dem Leder, umgewandelt werden. Der Vorgang wird um so irreversibler, je mehr ausgesprochener kolloidaler Natur das verwendete koagulierende Mittel ist, welches eine Absorptionsverbindung mit den Hautfasern eingeht. Tannin, Chromoxyd und andere Metalloxydhydrate, Anilinfarbstoffe geben die besseren Resultate (Zacharias).

Hydrosole der Metalloxyde und Metallsulfide sind auch vielfach untersucht worden. Ihre Eigenschaften bewegen sich zwischen beiden beschriebenen Systemen.

\$5. Verschiedene andere Eigenschaften der Kolloide können durch folgende Beispiele illustriert werden.
Die Leitfähigkeit der Sole zeigt großle Analogie mit der Leitfähigkeit der Suspensionen. Nach Quincke wandern in destilliertem Wasser suspendierte Stärkekörner, Metallteilchen, Quarz, Ton, Sanerstoff, Wasserstoff usw. an der Wand der umschliekenden Röhre im Sinne des positiven, in der Mitte derselben im Sinne des negativen Stromes. Indifferente Kolloide wandern meist zur Anode. Die so bei 110 Volt Spannung übergeführten Mengen sind sehr klein. Kolloidalgelöste Metalle, Salze, Sulfide, Zinnsäure, Molybdän- und Wolframblau sind negativ geladen; Eisenoxyd wandert zur Kathode, seinem basischen Charakter entsprechend. Ebenso sind positiv geladen Aluminiumbydroxyd und Oxyde der seltenen Erden. EiweiB ist in saurem Wasser elektropositiv, in alkalischem elektrogativ.

Wenn wir zu einer Zinnchloridlösung vorsichtig Ammoniak zusetzen, dann kommen wir zu sehr interessanten Ergebuissen (Kuriloff). Zuerst entsteht bekanntlich ein Niederschlag; welcher mit steigendem Ammoniakzusatz wächst: dann kommt ein Punkt, wo die ganze Fhüssigkeit gelatiniert, dann nimmt der Niederschlag wieder $a b$. In diesem Grenzgebiet erleidet der Ammoniakgehalt der Lösung einen plötzlichen Sprung. Die beiden Gebiete verhalten sich verschieden. In Oebiete des zunehmenden Niederschlages ist der Bodenkörper ein Oxychlorid von bestimmter Zusammensetzung, im zweiten Gebiet aber ist der Bodenkörper kolloidales Zinnoxyd. Die totale Koagulation im Orenzgebiet ist ein wichtiges Kennzeichen für den Uebergang von gewöhnlicher Lösung zur Pseudolösung.

Die kolloidalen Lösungen sind keine stabilen Gebilde. Der gelöste Körper erleidet stetige Aenderungen, die zur Zusammenflockung und Abscheidung desselben führen. Verschiedene Zusätze beschletnigen diesen Vorgang. Die Einflüsse sind aber spezifische Elektrolyte wirken an den meisten Soler gelbildend. Am schnellsten wirken Ionen auf entgegengesetzt geladene Teilchen. Im allgemeinen wirken H. und $\mathrm{OH}-$ Ionen verschieden. Hydroxylionen wirken sehr hãufig solbildend. Entgegengesetzt geladene Sole fällen einarider aus, ähnlich geladene nicht. Diese Fällung kann chemische Einwirkung vortäuschen 10). Das Maximum der Fällung geschieht unter bestimmten Verhältnissen "11) und bei groBem UeberschuB des einen Sols tritt häufig überhaupt keine Ausfällung ein.

in) Vergl. Duclaux C. R. 138, 144, 189.

i) W. Biltz. Ueber gegenseitige Beeinflussung kolloidal gelöster Stoffe. Ber. 37, 1095 . 
Bei der Fällung durch Salze läBt sich die Gesetzmäßigkeit beobachten, daB eine bestiminte Menge zur Kragulation der Lösung zugesetzt werden mub. Starke Säuren fällen bei viel geringerer Konzentration als schwache. Saize wirken proportional der Wertigkeit des Kations, also die der dreiwertigen Metalle am schnellsten. Die Wirkung der Elektrolyte ist nicht nur von der Konzentration abhängig, sondern auch von der Geschwindigkeit, nit welcher die Lösung des Elektrolyts zum Sole zugegeben wird (Frcundlich). Aher auch indifferente Stoffe wirken koagulierend. Alkohol koaguliert Gelaline, Abumin usw. Amylaikohol koaguliert Albumin mit Sicherheit bein gelinden Erwärmen, cbenso aus sauren wie aus alkalischen Flüssigkeiten (Zacharias), welche ani die Koagulation des Albumins hindernd wirken. Bei den organischen komplexen Kolloiden sind die Verhältnisse viel verwickeiter und lassen sich Regeln nicht so leicht antstellen wie bei den anorganischen Solen.

Es muB betont werden, dâ gewöhnliche Reaktionen beim Kolloidaizustand nicht vorkommen. Bestimmte chemisclie Eigenschaften können im latenten Zustand existieren, kommen aber zum Vorschein, sobald das Kolloidalgebilde zerstöıt wird. Die einzige Reaktion der Kolloide in diesem Sinne ist ihre Zersetzung. Kolloide sind also chemisch inert und reagieren chernisch blö, um zersetzt zu werden (Zacharias). Die Produkte der Zersetzung können kolloidaler Natur oder von molektularen Charakter und Dimensionen sein.

Eine weitere Folge ist dic, daß der Verteilungssatz auf das Absorptionsgleichgewicht nicht angewendet werden kam, weil er auf Molekularbetrachtungen gegründet ist und die experimentellen Ergebnisse durch diese Auffassung keine Deutung erfahren können. Das gilt auch für die Fälle, wo $"=1$ und die Absorptionsgleichung die äuBere form des Haag'schen Gesetzes $\frac{c}{c_{1}}=$ : $=$ a annimmt(Zacharias).

Aehnlich haben die Versuche gelehrt, daß Absorptionsgleichgewichte mit den Ergebnissen des Massenwirkungsgesetzes ${ }^{2}$ ) nicht stimmen

12) Uiese Tatsache läbt sich aus folgender eitnfachen Analogie erklären. Denken wir z. B, daB wir in einen Raum, in welchem sich die Dissoziationsprodukte $\mathrm{NH}_{3}$ und $\mathrm{H}_{8} \mathrm{~S}$ neben dem fesien Ammoniunlivdrosulfid befinden, gewölnnliche Kohle eintragen. Da die Kohle die Bestandtele des Gasgemenges NHo und $H 2 S$ fortdauernd absorbiert, kann man keine Konstanz des Dissoziationsdrucks erhaiten und das Massenwirkungsgesetz wird scheinbar seine An-
(Kuriloff) und sich auch in den Rahmen der von der Phasentegel 13) festgestellten Gleichgewichte nicht einordnen lassen (van Bemmelen, Kuriloff).

Wir möchten hier betonen, daß das Aufsuchen des Verhaltens der Teilungsverhältnisse bei der Absorption zu der Oleichung $\frac{c}{c_{1}{ }^{2}}=\alpha$ und zur Auffindung von Nebenwirkungen verhelfen kann. Sobald das Verhältnis $\frac{c}{c_{1}{ }^{r}}$ keine Konstante ist, muk man den Zustand sowohl des absorbierenden wie des absorbierten Körpers untersuchen. Bei der Absorption kann der absorbierende Körper entweder weiter koaguliert oder pepisisert werden; in ersten Falle nimmt sein Volum $a b$, im zweiten zu; zugleich ändert sich die Rigidität des Gels und seine inneren Kräfte. Wenn der absorbierte Körper kolloidal gelöst ist, dann gerinnt er und stört das $A b$ sorptionsgleichgewicht usw. (Zacharias).

Die zeitliche Aenderung der Eigenschaften ist schon von Graham an den Solen beobachtet worden, ist aber am besten in den kolloidalen Oxyden von van Bemmelen ${ }^{14}$ ) untersucht worden. Es hat sich gezeigt, daß durch die Zeit (sowohl als durch Druck und Erwärmung) nicht umkehrbare Modifikationen stattfinden, die mit Verlust des Wasserbindungsvermögens begleitet sind. Das nicht entwässerte Gel verliert mit der Zeit an Absorptionsvermögen. Viele Sole werden mit der Zeit trübe, was an vielen Farbstofflösungen beobachtet wird. EinfluB der Zeit auf die Fällung des Platinsols durch Elektrolyte ist auch von Freundlich beobachtet worden.

Schließlich ist die Abhängigkeit der Zusammensetzung von den äußeren Bedingungen das beste Merkmal des Kolloidalzustandes. Das aus einem Sol abgeschiedene Gel ist von verschiedenem Wassergenalt, je nach der Konzentration der ursprūnglichen Lösung. Die Zusammensetzung des Gels ist kontinuierlich abhängig von der Temperatur, von der Konzentration der flüssigen oder gasförmigen Phase und vom physikalischen Bau des Gels, der sich kontinuierlich modififizieren kann (van Bemmelen). Die Zusammensetrung einer Absorptionsverbindung ist abhängig

wendbarkeit verlieren. Auf ähnliche Weise erklären sich im Falle der Kolloide die Stönungen, die durch die Absoiption durth das Kolloidalgebilde von Bestandieilen der Gleichgewichtsflüssigkeit enstanden sind. 13) Denn der Begriff der Phase hat hier bereits eine andere Bedeutung.

i1) var Bemmelen. Die Absorption. 3. Abhavdlung. $z$. f. anorg. Chemie 1898, $98-146$. 
von der Konzentration des absorbierten Körpers in der Lösung, der Temperatur und verschiedenen Zusätzen. Eine Absorptionsverbindung existiert in allen Verhältnissen. Die besten Beispiele dieser Art sind die Verbindungen der Cellulose. Die gefärbte Faser und das Leder sind auch solche Absorptionsverbindungen (Zacharias).

Wir haben in der Abhandlung Gebrauch von der Bezeichnung Absorption gemacht. Viele Autoren gebrauchen die Bezeichnung Adsorption. Wie van Bemmeler 1900 betont hat, sind bei den Gelen die Wirkungen, die den Begriff der Absorption differenzieren, nicht voneinander zu trennen und Zacharias hat 1905 auf das Unstatthafte dies Begriffs Adsorption hingewiesen.

$\$ 6$. Indem wir nun glauben, durch obige Auseinandersetzungen gezeigt zu haben, daB die aufgestellten 12 Punkte über das ganze
Verhalten der Kolloide AufschluB geben und einem Diskussionsplan zugrunde gelegt werden közmen, möchten wir zum SchluB die spezifischen Reaktionen des Kolloidalzustandes kurz zusammenfassen (Zacharias). Das sind:

1. Quellung,

2. Solbildung,

3. Koagulation,

4. Absorption unter Bildung von Absorptionsverbindungen,

5. Zustandsänderung mit der Zeit,

6. Zersetzung unter Abbau des Kolloidalgebildes, entweder zu einfacheren Kolloidalgebilden oder zu Molekularverbindungen.

Es wird zu hoffen sein, daß bald die Gesetze dieser Reaktionen eine hestimmte Form annehmen, was in den Fällen, wo auch chemische Reaktionen primär oder sekundär mit auftreten, besonders wertvoll sein würde.

\title{
Ueber Kolloidchemie, mit besonderer Berücksichtigung der anorganischen Kolloide). . .
}

\author{
Von R. Zsigmondy.
}

Kolloide* nennt Graham diejenigen Stoffe, welche schwer durch eine Pergamentmembran diffundieren, zum Unterschied von den "Kristalloiden", bei denen Jas Gegenteil der Fall ist. Als Urbild der Kolloidsubstanzen wird der Leim hingestellt; Graham nennt die in Wasser gelösten Kolloide Hydrosole und die aus ihrien sich abscheidenden amorphen, häufig gallertartigen Körper Hydrogele; aus diesen entstehen durch Austausch des Wassers gegen Alkohol, konzentrierte Schwefelsäure etc. Alkogele, Sulfogele. Die Graham'sche Einteilung bezieht sich aber nicht auf einheitliche Stoffe, sofern man darunter einheitliche Substanzen (im Sinne des Chemikers) versteht, sondern vielmehr auf die Erscheinungen, welche auftreten, sobald zwei oder mehrere chemische individuen zueinander in innige Beziehungen getreten sind, wie sie in den Mischungen (als Lösung, Gallerten, Absorptionsverbindungen) vorliegen. Daher kann die Entscheidung über einige wichtige Fundamentalfragen der Kolloidchemie durch die ausschließliche Anwendung rein chemischer Methoden nicht getroffen werden,

") Auszug aus dem Vortrag geh. auf der Versammlung $D$. Naturforscher und Aerzte, Stuttgart, September 1906. - Ausführliche Darstellung wird demnächst bei Joh. Ambros. Barth, Leipzig, erscheinen. womit nicht gesagt sein soll, daA die Kolloidchemie nicht auch von der reinen Chemie große Fortschritte zu erwarten hätte. (Vgl. E. Fischer's Arbeiten tuber die Abbauprodukte des EiweiB, Erforschung der feinsten Strukturen der Hydrogele, die sich die Arbeiten von Nägeli, Bütschli, van Bemmelen u. a. zum Ziel genommen haben, ferner die Forschungen über die Natur der kolloidalen Lösungen.) Die einfachsten Beziehungen zwischen zweieriei Körpern liegen in den koiloidalen Lösungen vor. Zur năheren Charakterisierung dieser hat sich die Bestimimung des Molekulargewichtes als besonders nützlich erwiesen. Es ist sehr hoch und liegt zwischen 800 und 50000 , zuweilen erscheint es unbestimmt groB. Der diesen berechneten Werten zugrunde liegende kleine osmotische Druck kann auf die abnorme Molekülgröße in den Hydrosolen, wie auch darauf zurïckgefïhrt werden, daB ein Teil des gelösten Körpers oder seine Gesamtmasse in einen besonderen Zustand enthalten sind, in welchem sie keinen osmotischen Druck auszuüben vermögen. Mit dem Zurücktreten der osmotischen Volunenergie Hand in Hand geht das Auftreten einer optischen Inhomogenität. (Nachweis des Tyndall'schen Phänomens durch Picton und Linder, Bredig u. a., ultramikroskopiscine 